\title{
Asymptotic stability for a symmetric parabolic problem modeling Ohmic heating
}

\author{
Mingshu Fan' ${ }^{1}$, Anyin Xia² and Lei Zhang ${ }^{3 *}$
}

"Correspondence:

zhanglei@scu.edu.cn

${ }^{3}$ College of Computer Science,

Sichuan University, Chengdu,

610064, P.R. China

Full list of author information is

available at the end of the article

\begin{abstract}
We consider the asymptotic behavior of the solution of the non-local parabolic equation $u_{t}=(\kappa(u))_{r r}+\frac{(\kappa(u))_{r}}{r}+\frac{f(u)}{\left(a+2 \pi b \int_{0}^{1} f(u) r d r\right)^{2}}$, for $0<r<1, t>0$, with a

homogeneous Dirichlet boundary condition. The equation is the so-called Ohmic-heating model, which comes from thermal electricity in this paper, $u$ and $f(u)$ represent the temperature of the conductor and the electrical conductivity. The model prescribes the dimensionless temperature when the electric current flows through two axis-symmetric conductors, subject to a fixed electric potential difference. The global existence and uniform boundedness of the solution to the problem indicate that the temperature of the conductor remains uniformly bounded. Furthermore, the asymptotic stability of the global solution is obtained.
\end{abstract}

MSC: 35K20; 35K55; 35K65; 80M35

Keywords: Ohmic heating model; nonlocal parabolic equation; axis-symmetric; steady state; asymptotic stability

\section{Introduction}

The original motivation for studying the problem in this paper comes from the plasma Ohmic-heating process. The plasma is an electrical conductor and so it could be heated by passing a current through it. This is called Ohmic heating and it is the same kind of heating that occurs in thermistors.

First, we would like to address the work on the Ohmic-heating model with one conductor, which has been investigated intensively in [1-6] and references therein. The problem with only one conductor can be formulated in terms of the following problem with different boundary conditions:

$$
u_{t}=\Delta(\kappa(u))+\frac{\lambda f(u)}{\left(\int_{\Omega} f(u) d x\right)^{2}}, \quad \text { for } x \in \Omega, t>0 \text {, }
$$

where $\Omega \subset \mathbb{R}^{2}$ is an open, bounded domain, and $\kappa(u)$ is the diffusion function. $f(u)$ is the electrical conductivity and the parameter $\lambda$ is a positive constant, which depends upon the electric current or potential difference and also upon the 'size' of the conductor.

For the linear diffusion problem $\kappa(u)=u$, Lacey etc. have shown that the blow-up cannot take place if $f$ is an increasing function (see $[2,7]$ ). However, if $f$ is a decreasing function, with the aid of the comparison principle, global existence and asymptotic have been obtained for some special function $f$, such as $f(u)=e^{-u}$ in [2]. Taking advantage of this fact,

\section{第 Springer}

@2014 Fan et al.; licensee Springer. This is an Open Access article distributed under the terms of the Creative Commons Attribution License (http://creativecommons.org/licenses/by/2.0), which permits unrestricted use, distribution, and reproduction in any medium, provided the original work is properly cited. 


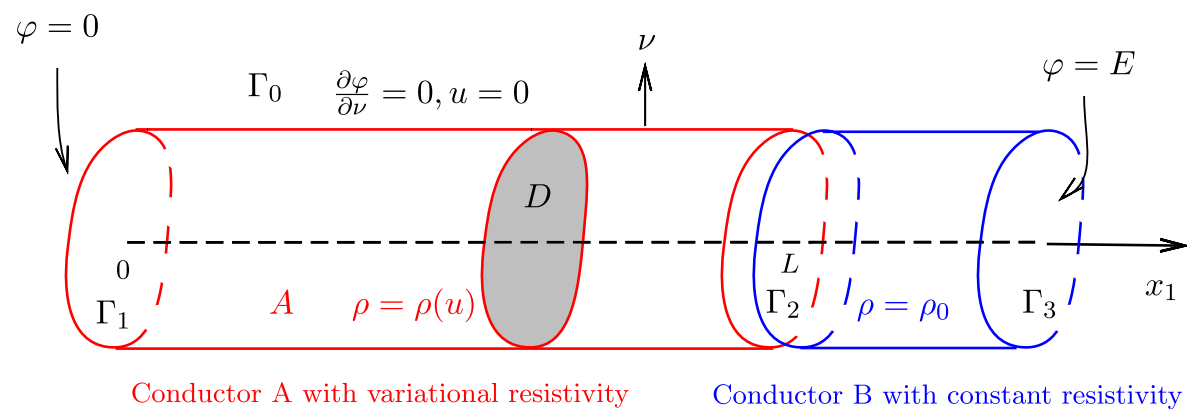

Figure 1 Electric current flows through two conductors.

Lacey $[2,3]$ and Tzanetis [4] proved the occurrence of blow-up for a one-dimensional model (1) and for the two-dimensional radially symmetric model (1).

However, to avoid the occurrence of the singularity in finite time, Lacey introduced an Ohmic-heating model with two conductors (see Figure 1). In the electric circuit, the conductor $A$ is in series with another conductor $B$ with constant electrical resistivity. Denote $u(x, t)$ and $\rho(u)=1 / f(u)$ for the temperature and the electrical resistivity of the conductor $A$, respectively. The global existence and asymptotic behavior have been shown for decreasing electrical conductivity function $f(u)$ in [8] by Du et al.

Furthermore, the problem with nonlinear diffusion has been investigated in [8] recently and similar results have been obtained. However, most previous results on asymptotic behavior we mentioned before are restricted to the one-dimensional case.

Here, we suppose that the conductor $A$ is a prismatic one, $L$ and $S$ represent its length and cross-sectional area, respectively, and the length of the conductor $B$ is $L^{\prime}$. Moreover, we assume that the diameter of the cross section $D$ is much less than $L$ and the temperature $u(x, t)$ is independent of the variable $x_{1}$. Suppose that the curved surface of the conductor $A, \Gamma_{0}$ is thermal, namely

$$
u=0 \quad \text { on } \Gamma_{0} .
$$

Based on the derivation in [2] (see also [4]), Du and Fan obtained the result that the temperature $u$ of the material satisfies the following problem with initial boundary-value conditions:

$$
u_{t}-\Delta(\kappa(u))=\frac{f(u)}{\left(a+b \int_{D} f(u) d x\right)^{2}}, \quad x \in D, t>0
$$

where $a=\frac{L}{E}, b=\frac{\rho_{0}}{E} L^{\prime}$ and $f(u)=\sigma(u)$.

When $f$ is decreasing, they proved that the comparison principle was valid and the solution of the model was always global in time. Furthermore, if $f$ is a decreasing exponential function, they proved that the solution of the problem converges asymptotically to the unique steady state. See also [9] and [10] for some results on the asymptotic behavior of the global solution in the one-dimensional case and axis-symmetry in the two-dimensional case for the linear diffusion problem $\kappa(u)=u$, respectively.

In this paper, we assume conductor $A$ is axis-symmetric, and we focus on the problem (2) in the radially symmetric case. So, if we assume additionally that the cross section $D$ be a 
unit disk and the initial data be radially and decreasing, i.e.

$$
u_{0}(x)=u_{0}(r) \quad \text { and } \quad u_{0}^{\prime}(r)<0 \text {, }
$$

where $r=|x| \in(0,1)$. Thus, the problem in the axis-symmetric case can be formulated in terms of the following non-local parabolic problem:

$$
\begin{cases}u_{t}=(\kappa(u))_{r r}+\frac{(\kappa(u))_{r}}{r}+\frac{f(u)}{\left(a+2 \pi b \int_{0}^{1} f(u) r d r\right)^{2}}, & \text { for } 0<r<1, t>0, \\ u(1, t)=u^{\prime}(0, t)=0, & \text { for } t>0, \\ u(r, 0)=u_{0}(r), & \text { for } 0 \leq r \leq 1,\end{cases}
$$

where $f$ is a continuous, positive, and decreasing function and the initial data $u_{0}(r)$ is a decreasing smooth positive function. The function $\kappa(s)$ satisfies

$$
\kappa(s) \in C^{2}\left(\mathbb{R}^{+}\right), \quad \kappa(s)>0, \quad \kappa^{\prime}(s)>0, \quad \kappa^{\prime \prime}(s)>0, \quad \text { for } s>0,
$$

and

$$
\kappa(0)=0 \text {. }
$$

The boundary condition $u(1, t)=0$ represents the temperature on the boundary $\Gamma_{0}$ is a constant, while $u_{r}(0, t)=0$ comes from the axis-symmetry of conductor $A$.

Moreover, it is easy to see that the axis-symmetric solution to the problem (3) is radially decreasing (see [11]).

Inspired by the work above, we would like to show the asymptotic stability of the problem (3) with the generally deceasing function $f(u)$.

Theorem 1.1 Assume that $f$ satisfies

$$
-s f^{\prime}(s)<f(s), \quad s>0
$$

the solution of the problem (3), $u(r, t)$, converges asymptotically to the unique steady state $\omega(r)$, namely,

$$
u(r, t) \rightarrow \omega(r), \quad \text { as } t \rightarrow+\infty
$$

for $0<r<1$.

Remark 1 The condition (6) is equivalent to $(s f(s))^{\prime}>0$, which indicates $f(s)$ is decreasing lower than $\frac{1}{s}$, such as $f(s)=s^{-p}, 0<p<1$. The condition (6) is structured to prove the uniqueness of the steady state, which was firstly structured by Tzanetis in [4].

Remark 2 When $f(s)=e^{-\kappa(s)}$, Du and Fan [8] have proved that the solution of the problem (3) converges asymptotically to the unique steady state. If $\kappa(s)$ additionally satisfies $s \kappa^{\prime}(s)<1$, then $e^{-\kappa(s)}$ is a special function of $f(s)$, which satisfies (6). 
Remark 3 The equations in models (1), (2), and (3) are quasi-linear parabolic equations with non-local sources. There are many works focusing on the global existence and blowup of non-local parabolic equations (see $[9,12-15]$ and the references therein).

The analysis and techniques in this paper are based on the analysis for the ordinary differential equations and on comparison arguments.

\section{The asymptotic stability for the problem (3)}

We start this section with introducing the comparison principle and the global existence of the solution to (3), which have been proved in [8].

Proposition 1 (Comparison principle) Suppose that $\bar{u}(r, t)$ satisfies

$$
\begin{cases}\bar{u}_{t}(r, t) \geq(\kappa(\bar{u}(x, t)))_{r r}+\frac{1}{r}(\kappa(u(r, t)))_{r}+\frac{f(\bar{u}(r, t))}{\left(a+b \int_{\Omega} f(\bar{u}(r, t)) d r\right)^{2}}, & 0<r<1, t>0, \\ \bar{u}(r, t) \geq 0, & r=1, t>0, \\ \bar{u}(r, 0) \geq u_{0}(r), & 0<r<1,\end{cases}
$$

and $\underline{u}(r, t)$ satisfies $(7)$ with the inequalities reversed, where $f(u)$ is monotonic decreasing and positive continuous function. Then $\bar{u}(r, t) \geq \underline{u}(r, t)$ for $0<r<1$.

Proposition 2 If $f(u)$ is a decreasing, positive, continuous function, then every nontrivial positive classical solution of (3) exists globally in time for any nontrivial positive bounded initial data. Furthermore, the solution $u(r, t)$ of (3) remains uniformly bounded.

First, we consider the steady-state problem corresponding to (3),

$$
\left\{\begin{array}{l}
(\kappa(\omega))_{r r}(r ; \mu)+\frac{1}{r}(\kappa(\omega))_{r}(r ; \mu)+\mu f(\omega(r ; \mu))=0, \quad 0<r<1 \\
\omega(1 ; \mu)=\omega_{r}(0 ; \mu)=0
\end{array}\right.
$$

with the positive parameter

$$
\mu=\frac{1}{\left(a+2 \pi b \int_{0}^{1} f(\omega(r ; \mu)) r d r\right)^{2}},
$$

and they give the uniqueness of the solution of the problem (8).

Moreover, under the condition (5), multiplying $r$ and $(\kappa(\omega))_{r}(r ; \mu)$ on both sides of the equation in (8), respectively, and integrating over $[0,1]$ yields

$$
\int_{0}^{1} f(\omega(r ; \mu)) r d r=-\frac{\left.(\kappa(\omega)(r ; \mu))_{r}\right|_{r=1}}{\mu}
$$

and

$$
\left(\left.(\kappa(\omega)(r ; \mu))_{r}\right|_{r=1}\right)^{2}=2 \mu \int_{0}^{\kappa(M(\mu))} f\left(\kappa^{-1}(s)\right) d s-2 \int_{0}^{1} \frac{\left((\kappa(\omega))_{r}(r ; \mu)\right)^{2}}{r} d r,
$$

where $M(\mu)=\omega(0 ; \mu)=\max _{r \in[0,1]} \omega(r ; \mu)$ and $\kappa^{-1}$ is the inverse function of $\kappa$. 
In view of the condition (4) and $\omega^{\prime}(r ; \mu)<0$ for any $r \in(0,1)$, it thus follows from (11) that

$$
\left.(\kappa(\omega)(r ; \mu))_{r}\right|_{r=1}=-\sqrt{2 \mu \int_{0}^{\kappa(M(\mu))} f\left(\kappa^{-1}(s)\right) d s-2 \int_{0}^{1} \frac{\left((\kappa(\omega))_{r}(r ; \mu)\right)^{2}}{r} d r .}
$$

Combining this with (9) and (10) yields

$$
\begin{aligned}
1 & =\mu\left(a+2 \pi b \int_{0}^{1} f(\omega(r ; \mu)) r d r\right)^{2} \\
& =a^{2} \mu+\frac{4 \pi^{2} b^{2}\left(\left.(\kappa(\omega)(r ; \mu))_{r}\right|_{r=1}\right)^{2}}{\mu}-\left.4 \pi a b(\kappa(\omega)(r ; \mu))_{r}\right|_{r=1} .
\end{aligned}
$$

To show the uniqueness of the initial value problem (8) with (9), it suffices to show that there exists a unique root $\mu \in\left(0, \frac{1}{a^{2}}\right)$ of equation (13).

Define

$$
F(\mu):=a^{2} \mu+\frac{4 \pi^{2} b^{2}\left(\left.(\kappa(\omega)(r ; \mu))_{r}\right|_{r=1}\right)^{2}}{\mu}-\left.4 \pi a b(\kappa(\omega)(r ; \mu))_{r}\right|_{r=1}-1, \quad \text { for } \mu>0 .
$$

It is easy to see that the problem (8) does not possess nontrivial solution with the parameter $\mu=0$. That is, $\omega \equiv 0$ and $M=0$ for $\mu=0$. Then multiplying $\frac{1}{\mu}$ on both sides of (11) and taking the limit $\mu \rightarrow 0^{+}$yield

$$
\lim _{\mu \rightarrow 0^{+}} \frac{\left(\left.(\kappa(\omega)(r ; \mu))_{r}\right|_{r=1}\right)^{2}}{\mu}=0
$$

since

$$
\frac{\left(\left.(\kappa(\omega)(r ; \mu))_{r}\right|_{r=1}\right)^{2}}{\mu} \text { and } \int_{0}^{1} \frac{\left((\kappa(\omega))_{r}(r ; \mu)\right)^{2}}{r} d r
$$

are both positive and

$$
\lim _{\mu \rightarrow 0^{+}} \int_{0}^{\kappa(M(\mu))} f\left(\kappa^{-1}(s)\right) d s=0
$$

Thus, $F(0+)<0$. In view of $F\left(1 / a^{2}\right)>0$, there exists at least one root $\mu \in\left(0,1 / a^{2}\right)$ to equation (13).

Due to the condition $\omega_{r}(0 ; \mu)=0$, we can take an odd extension to the problem (8), and formulate the following problem:

$$
\left\{\begin{array}{l}
(\kappa(\omega))_{r r}(r ; \mu)+\frac{1}{r}(\kappa(\omega))_{r}(r ; \mu)+\mu f(\omega(r ; \mu))=0, \quad-1<r<1, \\
\omega( \pm 1 ; \mu)=0 .
\end{array}\right.
$$

We claim that the steady solution $\omega(r ; \mu)$ is increasing strictly with respect to the parameter $\mu$, and it is always positive, namely, $\omega_{\mu}(r ; \mu)>0$, for any $0 \leq r \leq 1$. In fact, differentiating on both sides of equation (14) with respect to the parameter $\mu$ gives

$$
-(\kappa(\omega))_{\mu r r}(r ; \mu)-\frac{1}{r}(\kappa(\omega))_{\mu r}(r ; \mu)-\frac{\mu f^{\prime}(\omega(r ; \mu))}{\kappa^{\prime}(\omega(r ; \mu))}(\kappa(\omega))_{\mu}(r ; \mu)=f(\omega(r ; \mu))>0 .
$$


In view of $(\kappa(\omega))_{\mu}( \pm 1 ; \mu)=0$, it follows from the standard comparison arguments to equation (15) that we have $(\kappa(\omega))_{\mu}(r ; \mu)>0$, for any $r \in[-1,1]$. Then $(\kappa(M))_{\mu}(\mu)=$ $(\kappa(\omega))_{\mu}(0 ; \mu)>0$, and $\omega_{\mu}(r ; \mu)>0$, for any $r \in[-1,1]$, since $\kappa^{\prime}(s)>0$ for all $s>0$.

For convenience, we set

$$
\varphi(\mu):=\frac{\left(\left.(\kappa(\omega)(r ; \mu))_{r}\right|_{r=1}\right)^{2}}{\mu}=\frac{2 \mu \int_{0}^{\kappa(M(\mu))} f\left(\kappa^{-1}(s)\right) d s-2 \int_{0}^{1} \frac{\left((\kappa(\omega))_{r}(r ; \mu)\right)^{2}}{r} d r}{\mu} .
$$

Then, from (12), we have

$$
F(\mu)=a^{2} \mu+4 \pi^{2} b^{2} \varphi(\mu)+4 \pi a b \sqrt{\mu} \sqrt{\varphi(\mu)}-1,
$$

where we have used the fact $\left.(\kappa(\omega)(r ; \mu))_{r}\right|_{r=1}<0$.

Denote $\alpha=\sqrt{\mu}, z\left(r ; \alpha^{2}\right)=\frac{\kappa(\omega)(r ; \mu)}{\alpha}=\frac{\kappa(\omega)\left(r ; \alpha^{2}\right)}{\alpha}$; we can rewrite

$$
\varphi(\mu)=\left(z_{r}\left(1 ; \alpha^{2}\right)\right)^{2}
$$

Thanks to (5) and the boundary conditions of (8), by a direct computation, we have $z_{\alpha}\left(1 ; \alpha^{2}\right)=z_{\alpha r}\left(0 ; \alpha^{2}\right)=z\left(1 ; \alpha^{2}\right)=z_{r}\left(0 ; \alpha^{2}\right)$ and

$$
z_{r r}\left(r ; \alpha^{2}\right)+\frac{z_{r}\left(r ; \alpha^{2}\right)}{r}+\alpha f\left(\omega\left(r ; \alpha^{2}\right)\right)=0
$$

Differentiating on both sides of (17) with respect to the parameter $\alpha$, we have

$$
\begin{gathered}
z_{\alpha r r}\left(r ; \alpha^{2}\right)+\frac{z_{\alpha r}\left(r ; \alpha^{2}\right)}{r}+\alpha^{2} f^{\prime}\left(\omega\left(r ; \alpha^{2}\right)\right) z_{\alpha}\left(r ; \alpha^{2}\right) \\
=-f\left(\omega\left(r ; \alpha^{2}\right)\right)-f^{\prime}\left(\omega\left(r ; \alpha^{2}\right)\right) \omega\left(r ; \alpha^{2}\right)<0,
\end{gathered}
$$

where we have used the crucial assumption (6). By the maximum principle and Hopf's boundary lemma, we have

$$
z_{\alpha}\left(r ; \alpha^{2}\right)>0 \text { and } z_{\alpha r}\left(1 ; \alpha^{2}\right)<0 \text {. }
$$

Thus,

$$
\varphi^{\prime}(\mu)=\frac{1}{2 \alpha} \frac{d}{d \alpha}\left(z_{r}\left(1 ; \alpha^{2}\right)\right)^{2}>0
$$

since $z_{r}\left(1 ; \alpha^{2}\right)=\frac{\left.\left(\kappa(\omega)\left(r ; \alpha^{2}\right)\right)_{r}\right|_{r=1}}{\alpha}<0$.

As a result of the above, by equation (16), we obtain $F^{\prime}(\mu)>0$, for any $\mu \in\left(0,1 / a^{2}\right)$. Furthermore, equation (13) possesses a unique root $\mu^{*}$ in $\left(0,1 / a^{2}\right)$, which shows that the problem possesses a unique steady state $\omega\left(r, \mu^{*}\right)$.

Next, we will show that the global solution of the problem (3) converges to its steady state. Inspired by the form of the steady state $\omega\left(r ; \mu^{*}\right)$, we seek for the upper solution decreasing in time of the form $\bar{v}(r, t)=\omega(r ; \bar{\mu}(t))=\bar{\omega}$, where the smooth function $\bar{\mu}(t)$ will be determined later. 
A series of computations yields

$$
\begin{aligned}
\bar{v}_{t} & -(\kappa(\bar{v}))_{r r}-\frac{1}{r}(\kappa(\bar{v}))_{r}-\frac{f(\bar{v})}{\left(a+2 \pi b \int_{0}^{1} r f(\bar{v}) d r\right)^{2}} \\
& =\bar{\omega}_{\bar{\mu}} \cdot \bar{\mu}^{\prime}(t)-(\kappa(\bar{\omega}))_{r r}-\frac{1}{r}(\kappa(\bar{\omega}))_{r}-\frac{f(\bar{\omega})}{\left(a+2 \pi b \int_{0}^{1} r f(\bar{\omega}) d r\right)^{2}} \\
& =\bar{\omega}_{\bar{\mu}} \cdot \bar{\mu}^{\prime}(t)-\frac{f(\bar{\omega})}{\left(a+2 \pi b \int_{0}^{1} r f(\bar{\omega}) d r\right)^{2}}\left[1-\bar{\mu}(t)\left(a+2 \pi b \int_{0}^{1} r f(\bar{\omega}) d r\right)^{2}\right] \\
& =\bar{\omega}_{\bar{\mu}}\left\{\bar{\mu}^{\prime}(t)-\frac{f(\bar{\omega})}{\bar{\omega}_{\bar{\mu}}\left(a+2 \pi b \int_{0}^{1} r f(\bar{\omega}) d r\right)^{2}}\left[1-\bar{\mu}(t)\left(a+2 \pi b \int_{0}^{1} r f(\bar{\omega}) d r\right)^{2}\right]\right\} .
\end{aligned}
$$

We will seek a function $\bar{\mu}(t)$ such that the right hand side of (18) is nonnegative for $0<r<1$ and $t>0$. Since $u_{0}(r)$ and $u_{0}^{\prime}(r)$ are bounded in $[0,1]$, we can choose $\bar{\mu}(0)$, such that

$$
\bar{v}(r, 0)=\omega(r, \bar{\mu}(0)) \geq u_{0}(r) .
$$

Set

$$
g(\bar{\mu}(t))=\left(\inf _{r \in[0,1]} \frac{f(\bar{\omega})}{\bar{\omega}_{\bar{\mu}}}\right) \frac{1}{\left(a+2 \pi b \int_{0}^{1} r f(\bar{\omega}) d r\right)^{2}}\left[\bar{\mu}(t)\left(a+2 \pi b \int_{0}^{1} r f(\bar{\omega}) d r\right)^{2}-1\right] .
$$

Note that $F(\mu)$ is increasing with respect to $\mu$, then $g(\bar{\mu}(t)) \geq 0$, provided that $\bar{\mu}(t) \geq \mu^{*}$, where $\mu^{*}$ is the unique root of (13). Thus, we can choose a decreasing function $\bar{\mu}(t)$ such that

$$
0 \geq \bar{\mu}^{\prime}(t) \geq-g(\bar{\mu}(t)) \text { and } \lim _{t \rightarrow \infty} \bar{\mu}(t)=\mu^{*}
$$

Then we have established an upper solution $\bar{v}(r, t)=\omega(r ; \bar{\mu}(t))$ to the problem (3), which converges to the steady state, i.e.

$$
\bar{v}(r, t) \rightarrow \omega\left(r ; \mu^{*}\right)=\omega(r), \quad \text { as } t \rightarrow \infty .
$$

Similarly, we can choose an increasing function $\underline{\mu}(t)$, which tends to $\mu^{*}$, as $t \rightarrow \infty$, and we construct a lower solution as $\underline{v}(r, t)=\omega(r ; \underline{\mu}(t))$. Finally, by the comparison principle, we obtain a pair of upper-lower solutions $(\bar{v}(r, t), \underline{v}(r, t))$, such that

$$
\underline{v}(r, t) \leq u(r, t) \leq \bar{v}(r, t), \quad \text { for } r \in[0,1] \text { and } t>0,
$$

and taking the limit $t \rightarrow \infty$, it completes the proof of Theorem 1.1.

Competing interests

The authors declare that they have no competing interests. 


\section{Author details}

${ }^{1}$ College of Mathematics, Southwest Jiaotong University, Chengdu, 610031, P.R. China. ${ }^{2}$ School of Mathematics and Computer Engineering, Xihua University, Chengdu, 610039, P.R. China. ${ }^{3}$ College of Computer Science, Sichuan University, Chengdu, 610064, P.R. China.

\section{Acknowledgements}

The authors would like to thank the referees for careful reading. This work is supported in part by an NSFC grant.

Received: 7 January 2014 Accepted: 29 January 2014 Published: 14 Feb 2014

\section{References}

1. Kavallaris, NI, Nadzieja, T: On the blow-up of the thermistor problem. Proc. Edinb. Math. Soc. 50, 389-409 (2007)

2. Lacey, AA: Thermal runaway in a non-local problem modelling Ohmic heating. Part I: Model derivation and some special cases. Eur. J. Appl. Math. 6, 127-144 (1995)

3. Lacey, AA: Thermal runaway in a non-local problem modelling Ohmic heating. Part II: General proof of blow-up and asymptotics of runaway. Eur. J. Appl. Math. 6, 201-224 (1995)

4. Tzanetis, DE: Blow-up of radially symmetric solutions of a nonlocal problem modelling Ohmic heating. Electron. J. Differ. Equ. 2002(11), 1-26 (2002)

5. Tzanetis, DE, Vlamos, PM: A nonlocal problem modelling Ohmic heating with variable thermal conductivity. Nonlinear Anal., Real World Appl. 2(4), 443-454 (2001)

6. Tzanetis, DE, Vlamos, PM: Some interesting special cases of a nonlocal problem modelling Ohmic heating with variable thermal conductivity. Proc. Edinb. Math. Soc. 44, 585-595 (2001)

7. Bebernes, JW, Lacey, AA: Global existence and finite-time blow-up for a class of non-local parabolic problem. Adv. Differ. Equ. 2, 827-841 (1997)

8. Du, LL, Fan, MS: Thermal runaway for a nonlinear diffusion model in thermal electricity. Discrete Contin. Dyn. Syst., Ser. A 33, 2349-2368 (2013)

9. Fan, MS, Du, LL: Asymptotic behavior for an Ohmic heating model in thermal electricity. Appl. Math. Comput. 218, 10906-10913 (2012)

10. Xia, A, Fan, M, Li, S: Asymptotic stability for an axis-symmetric Ohmic heating model in thermal electricity. J. Appl. Math. 2013, Article ID 387565 (2013)

11. Gidas, B, Ni, WM, Nirenberg, L: Symmetry and related properties via the maximum principle. Commun. Math. Phys. 68, 209-243 (1979)

12. Du, LL: Blow-up for a degenerate reaction-diffusion system with nonlinear nonlocal sources. J. Comput. Appl. Math 202, 237-247 (2007)

13. $\mathrm{Du}, \mathrm{LL}, \mathrm{Mu}, \mathrm{CL}$, Fan, MS: Global existence and non-existence for a quasilinear degenerate parabolic system with non-local source. Dyn. Syst. 20, 401-412 (2005)

14. Du, LL, Yao, ZA: Localization of blow-up points for a nonlinear nonlocal porous medium equation. Commun. Pure Appl. Anal. 6, 183-190 (2007)

15. Fan, MS, Mu, CL, Du, LL: Uniform blow-up profile for a nonlocal degenerate parabolic system. Appl. Math. Sci. 1, 13-23 (2007)

10.1186/1687-2770-2014-40

Cite this article as: Fan et al.: Asymptotic stability for a symmetric parabolic problem modeling Ohmic heating.

Boundary Value Problems 2014, 2014:40

\section{Submit your manuscript to a SpringerOpen ${ }^{\circ}$ journal and benefit from:}

- Convenient online submission

- Rigorous peer review

- Immediate publication on acceptance

- Open access: articles freely available online

- High visibility within the field

- Retaining the copyright to your article 